NSF-ITP-96-150

\title{
Black hole pairs and supergravity domain walls
}

\author{
A. Chamblin \\ Institute for Theoretical Physics, \\ University of California, \\ Santa Barbara, California 93106-4030, U.S.A. \\ and \\ J. M. A. Ashbourn-Chamblin \\ Wolfson College, University of Oxford, \\ Oxford OX2 6UD, England
}

\begin{abstract}
We examine the pair creation of black holes in the presence of supergravity domain walls with broken and unbroken supersymmetry. We show that black holes will be nucleated in the presence of non-extreme, repulsive walls which break the supersymmetry, but that as one allows the parameter measuring deviation from extremality to approach zero the rate of creation will be suppressed. In particular, we show that the probability for creation of black holes in the presence of an extreme domain wall is identically zero, even though an extreme domain wall has repulsive gravitational energy. This is consistent with the fact that the supersymmetric, extreme domain wall configurations are BPS states and should be stable against quantum corrections. We discuss how these walls arise in string theory, and speculate about what string theory might tell us about these objects.
\end{abstract}

PACS: 04.70.Dy; 04.60.-m; 11.27.+d; 04.65.+e; 12.60.J

Keywords: Black hole pair production; Domain walls; Supergravity 


\section{Introduction}

Recently, there has been considerable interest in the study of domain walls which arise in $N=1$ supergravity (SUGRA) theories. When the dilatonic coupling is turned off, these walls correspond to boundaries between regions of isolated vacua of a $N=1$ supergravity matter potential. Such domain walls and their global spacetime structure are extensively analysed in recent literature $([1],[2],[3],[4],[5]$, [6], [8]). A common feature of many of these solutions is that the walls are repulsive, i.e., inertial observers perceive the wall as a negative energy gravitational source. Therefore, in analogy with the arguments put forth in [7], we expect these SUGRA configurations to be unstable to quantum tunneling events such as black hole pair production, and indeed this is what we find in this paper.

Since we shall be interested in effects which arise because of the global gravitational properties of these solutions, we will only sketch here the relevant aspects of the configurations and refer the reader to the literature for more detail.

With this in mind, we begin by reiterating that (for now at least) we have turned off the dilaton coupling. It turns out [1] that there are three basic species of SUGRA domain walls, which can be described as follows:

i) Extreme domain walls, which interpolate between isolated supersymmetric minima.

ii) Non-extreme walls, which are expanding 'two-centred' bubbles.

iii) Ultra-extreme walls, which are simply false vacuum bubbles.

Within each of these phyla of walls, there are sub-classifications which specify more detailed properties of the solutions. 


\section{Extreme Walls}

First, we describe the different types of extreme vacuum domain walls. By construction [1], these extreme walls are static, planar and have a fixed energy density

$$
\sigma=\sigma_{\text {ext }}
$$

which is determined by the values of the cosmological constant on each side of the wall ( $\sigma_{\text {ext }}$ is the 'extremal' value of the energy density of the wall, i.e., $\sigma_{\text {ext }}$ is a Bogomol'nyi-type bound which extreme walls saturate [1]). Now, it turns out ([3], [4]) that without loss of generality we can take the extreme wall configurations to be conformally flat, i.e., there exists a conformal factor $A(z)$ (where ' $z$ ' is the normal direction to the wall) so that the metric may be written

$$
d s^{2}=A(z)\left(d t^{2}-d z^{2}-d x^{2}-d y^{2}\right)
$$

With this ansatz, the extreme vacuum walls can then be classified in terms of how the conformal factor $A(z)$ behaves on each side of the wall. It turns out that $A(z)$ has three basic 'signatures', each of which corresponds to a distinct 'type' of domain wall, as outlined:

Type I: A Type I extreme vacuum wall interpolates between a SUSY anti-de Sitter (adS) vacuum and a Minkowski SUSY vacuum. On the Minkowski side $(z<0)$, the conformal factor is constant

$$
A(z)=1, z<0
$$

whereas on the adS side, the conformal factor goes like $\frac{1}{z^{2}}$ :

$$
A(z) \longrightarrow \frac{3}{|\Lambda| z^{2}}, z \longrightarrow+\infty
$$

where $\Lambda$ is the cosmological constant. The surface energy density of the wall is then determined to be

$$
\sigma_{\mathrm{ext}}=\frac{1}{4 \pi \sqrt{3}} \sqrt{|\Lambda|}
$$


(we are working in units for which $G=c=\hbar=1$ ). Visually, the metric conformal factor looks as is illustrated below:

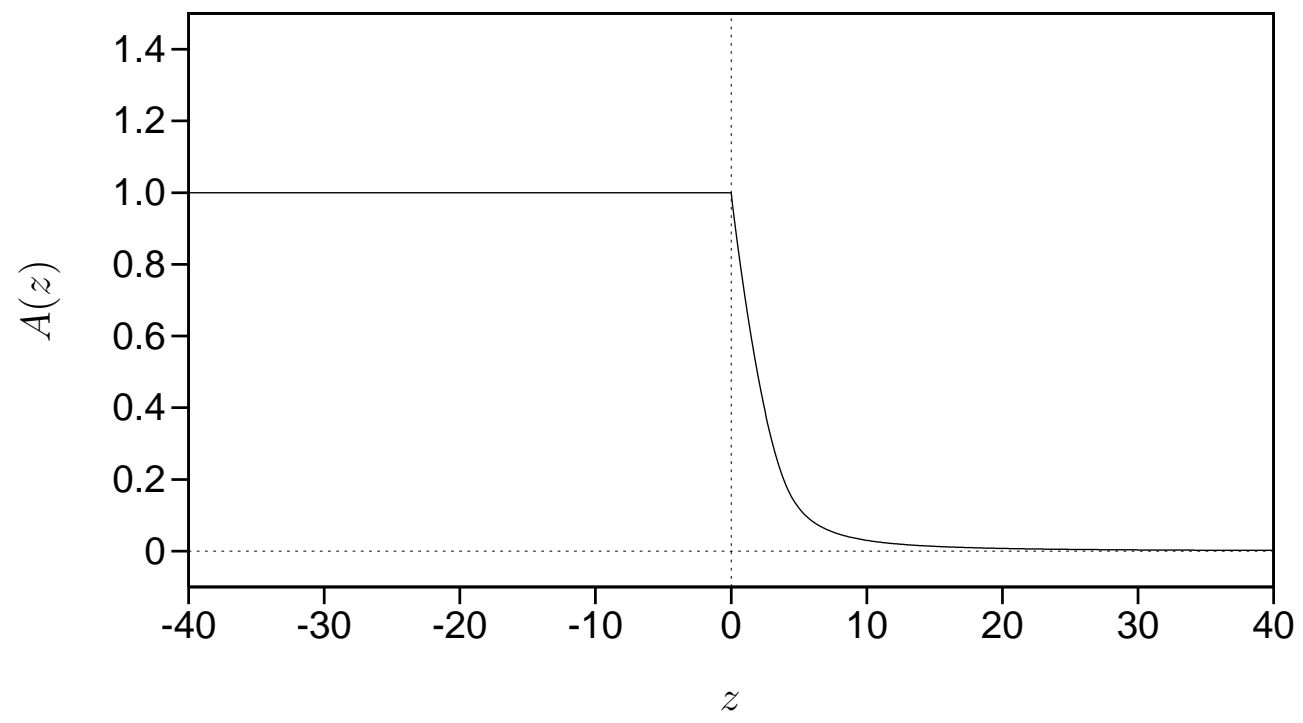

Type II: A Type II extreme vacuum wall interpolates between two SUSY adS vacua, such that the conformal factor has the same asymptotic behaviour on each side of the wall,

$$
A(z) \longrightarrow \frac{3}{\left|\Lambda_{ \pm}\right| z^{2}}, z \longrightarrow \pm \infty
$$

where $\Lambda_{+}$is the cosmological constant for $z>0$ and $\Lambda_{-}$for $z<0$ (i.e., $\Lambda_{+}$is not necessarily equal to $\Lambda_{-}$). The energy density of a Type II wall is calculated to be

$$
\sigma_{\mathrm{ext}}=\frac{1}{4 \pi \sqrt{3}}\left(\sqrt{\left|\Lambda_{-}\right|}+\sqrt{\left|\Lambda_{+}\right|}\right)
$$

Type III: A Type III extreme vacuum wall again interpolates between two SUSY adS vacua, only now the conformal factor has different asymptotic behaviour on each side of the wall. Namely, on one side of the wall ( $z>0$, say), $A(z)$ falls off as usual:

$$
A(z) \longrightarrow \frac{3}{\left|\Lambda_{+}\right| z^{2}}, z \longrightarrow+\infty
$$

whereas on the other side $A(z)$ blows up as $z$ approaches some critical value $z_{c}$ :

$$
A(z) \longrightarrow \frac{3}{\left|\Lambda_{-}\right|\left(z-Z_{c}\right)^{2}}, z \longrightarrow z_{c}
$$


The energy density is then given as

$$
\sigma_{\text {ext }}=\frac{1}{4 \pi \sqrt{3}}\left|\sqrt{\left|\Lambda_{-}\right|}-\sqrt{\left|\Lambda_{+}\right|}\right|
$$

The point $z_{c}$ is an infinite spacelike (proper) distance away from all other points.

In order to analyse geodesic motion in these extreme wall backgrounds, the simplest thing to do is just recover the Lagrangian of motion from the metric via the action principle:

$$
L=A(z)\left(\dot{t}^{2}-\dot{z}^{2}-\dot{x}^{2}-\dot{y}^{2}\right)
$$

Clearly, no force is felt in either the $x$ or $y$ directions (since $A$ only depends on $z$ ), and so only the $t$ and $z$ terms yield non-trivial motion. Introducing the energy per mass term $\varepsilon=A \dot{t}$, one integrates Equation (2) [10] for timelike particles $(L=1)$ to obtain the equation of motion

$$
z^{2}-t^{2}=\frac{3}{\left|\Lambda_{ \pm}\right| \varepsilon^{2}}
$$

Equation (3) with $\Lambda_{+}$applies in the region $z>0$, and likewise for the region $z<0$.

From (3), it is easy to see that on the Minkowski side of a Type I wall, freely falling massive test particles experience no gravitational force. However, an inertial observer on the adS side of a Type I or Type II domain wall is clearly moving on a hyperbola of acceleration away from the wall. Therefore, in order to remain a fixed distance $z_{0}$ from the wall, an observer would have to turn on the rocket boosters and accelerate towards the wall. In order to pass through the wall from the adS side, the observer would need an initial velocity of at least $\sqrt{1-A\left(z_{0}\right)}$. Observers who start out from $z_{0}$ with less than this velocity will be repulsed.

Thus, both Type I and Type II extremal walls exhibit repulsive behaviour, and we would naively expect this repulsive energy to contribute to the nucleation of black hole pairs. However, as we shall argue later, the rate of creation in these supersymmetric backgrounds is exactly zero, essentially because the action drops to minus infinity very quickly as extremality is approached. As for Type III walls, although they also have repulsive properties, they will not be considered in this paper, for the simple reason that the side of a Type III wall where the conformal 
factor diverges is limited by a timelike affine boundary. However, no domain wallblack hole configurations have such a boundary condition, and so the Type III walls are a priori not involved with the issue of black hole pair creation.

\section{Non-extreme Walls}

As was pointed out in [1], extreme walls bound SUSY vacua such that the gravitational potentials of the combined vacua exactly counterbalance the repulsive gravitational potential of the wall; the result of this exact balance is of course the fact that the wall is actually a static, timelike plane with an induced metric isometric to $2+1$ Minkowski space. When we perturb $\sigma$ away from $\sigma_{\text {ext }}$, and break supersymmetry, we expect the wall to become non-static.

Here, we describe the case where $\sigma>\sigma_{\text {ext }}$, the non-extreme domain walls. Since increasing the energy density of the walls increases the repulsive gravitational energy, we still expect these non-extreme walls to repel inertial observers, and indeed this is what happens. In fact, the non-extreme walls are simply another example of the standard 'two-centred' bubbles which often appear as domain wall solutions. The standard vacuum domain wall with $\Lambda=0$ on each side [12] is such a two-sided bubble; it is known as the 'Vilenkin-Ipser-Sikivie' (VIS) solution. In [7] we showed that black hole pairs will be nucleated in the background of VIS, and so it is hardly surprising that a similar thing occurs when we turn on a cosmological constant on each side of the bubble. In fact, a similar construction was considered in a recent paper of Mann [11], although he is still using VIS walls to nucleate the holes (i.e., the cosmological constant is coming from a 3-form field strength).

In more detail, the two-sided bubble is described by taking two regions of adS and glueing them together along a common timelike boundary homeomorphic to $S^{2} \times \mathbb{R}$. The boundary along which they are joined is chosen to satisfy the Israel

matching conditions [12]. Intuitively, to an observer on either side of the bubble, a non-extreme domain wall is a sphere which accelerates uniformly away from them in adS space. 
More explicitly, let $\Lambda_{1}$ be the cosmological constant on one side of the wall and $\Lambda_{2}$ the value on the other. Assume that on each side of the wall the spacetime metric assumes the form [1]:

$$
d s^{2}=e^{2 a(z)}\left\{d t^{2}-d z^{2}-S^{2}(t)\left[\left(1-\chi r^{2}\right)^{-1} d r^{2}+r^{2} d \phi^{2}\right]\right\}
$$

where

$$
S(t)=\left\{\begin{array}{cl}
1, & \chi=0 \\
\cosh \beta t, & \chi=\beta^{2}
\end{array}\right.
$$

$\beta>0$ a constant. Then the vacuum Einstein equations reduce to

$$
\dot{a}^{2}-\beta^{2}=e^{2 a}\left(\frac{-\Lambda}{3}\right)
$$

When $\Lambda<0$ the solution to (4) is

$$
a(z)=-\ln \left\{\beta \sinh \left(\beta z-\beta z^{\prime}\right) \sqrt{\frac{3}{-\Lambda}}\right\}
$$

The energy density of such a non-extreme wall is calculated to be

$$
\sigma=\frac{1}{4 \pi}\left(\frac{-\Lambda_{1}}{3}+\beta^{2}\right)^{\frac{1}{2}}+\frac{1}{4 \pi}\left(\frac{-\Lambda_{2}}{3}+\beta^{2}\right)^{\frac{1}{2}}
$$

We will begin the next section by considering the pair production of black holes in the presence of these non-extreme walls, since these solutions are the most obvious generalisations of the work described in [7].

\section{Ultra-extreme Walls}

Since ultra-extreme walls have $\sigma<\sigma_{\text {ext }}$, we again expect them to be non-static and in fact we expect them to have attractive gravitational energy. Indeed, this turns out to be the case and so these walls cannot be unstable to tunneling phenomena such as black hole pair production. We will therefore have nothing more to say about ultra-extreme walls. 


\section{Pair Production of Black Holes by Non-extreme Vacuum Walls}

In this section we shall be strictly concerned with the pair creation of black holes carrying a single $U(1)$ charge, i.e., Reissner-Nordström anti-de Sitter (RNadS) holes. Of course, we could also consider holes which are coupled to a dilaton field, since charged dilaton black holes (with $\Lambda<0$ ) which are asymptotically adS are known to exist [13]; however, we shall not consider such complications in this paper.

Thus, we seek timelike three-surfaces, which satisfy the Israel matching conditions, in the RNadS solution, which is most conveniently written as

$$
d s^{2}=-f(r) d t^{2}+\frac{d r^{2}}{f(r)}+r^{2} d \Omega^{2}
$$

where $d \Omega^{2}$ is the standard round metric on $S^{2}$ and

$$
f(r)=1-\frac{2 m}{r}+\frac{q^{2}}{r^{2}}-\frac{\Lambda}{3} r^{2}
$$

Of course, the equation of motion for domain walls in an arbitrary (spherically symmetric) black hole background is well-known [7], [11]. It is given as

$$
\sqrt{f(r)-\dot{r}^{2}}=2 \pi \sigma r
$$

where . $\cong f^{-\frac{1}{2}} \partial_{t}$. That is, (7) determines the radial motion of a non-extreme spherical wall surrounding a RNadS black hole.

We are particularly interested in this wall motion on the Euclidean section of RNadS. As in [7], we define the domain wall period, $\beta_{W}$, to be the amount of 'imaginary time' $\tau$ it takes the domain wall to interpolate between its minimal radius, $r_{\min }$, and its maximal radius, $r_{\max }$, on the instanton:

$$
\beta_{W}=\oint_{r_{\min }}^{r_{\max }} d \tau=\oint_{r_{\min }}^{r_{\max }} \frac{d r}{\sqrt{f\left(f-(2 \pi \sigma r)^{2}\right)}}
$$

i.e., $r_{\min }$ and $r_{\max }$ are the turning points where $\dot{r}=0$.

As in [7], we now posit the consistency condition, that in order for the domain wall motion to correspond to a well-defined Euclidean section, the wall must not 
intersect itself. That is to say, the domain wall period $\left(\beta_{W}\right)$ must be an integer submultiple of the period of the Euclidean section of RNadS (denoted $\left.\beta_{R N}\right)$ :

$$
\beta_{W}=\frac{\beta_{R N}}{n}, n \in \mathbb{Z}_{+}
$$

Also, there is the obvious requirement that $r_{\min }$ and $r_{\max }$ both be positive and real, and this condition leads to a bound on the mass: $\frac{1}{3 \sqrt{3} \beta} \leq m \leq \frac{1}{4 \beta}$.

As was pointed out in [14], in order for the RNadS metric (5) to describe a charged black hole in an asymptotically adS space with a non-degenerate horizon, the quartic $r^{2} f(r)$ must have a simple root $r_{0}>0$ such that $f(r)>0$ for all $r>r_{0}$. This can occur if and only if the mass satisfies an inequality

$$
m>m_{c}(q)
$$

where

$$
m_{c}(q)=\frac{l}{3 \sqrt{6}}\left(\sqrt{1+12\left(\frac{q}{l}\right)^{2}}+2\right)\left(\sqrt{1+12\left(\frac{q}{l}\right)^{2}}-1\right)^{\frac{1}{2}}
$$

where $\Lambda=\frac{-3}{l^{2}}$ as always. Since we desire to create holes with non-degenerate horizons, we will assume this inequality throughout. When $q=0, r_{h}=$ 'horizon radius' is defined as the positive solution of $f(r)=0$. When $q \neq 0, r_{h}$ is defined as the largest positive solution of $f(r)=0$. For fixed $q$, then, $r_{h}$ is a monotone increasing function of $m$ :

$$
r_{c}<r_{h}(m, q)<\infty \quad \text { as } \quad m_{c}<m<\infty
$$

where

$$
r_{c}(q)=\frac{l}{\sqrt{6}}\left(\sqrt{1+12\left(\frac{q}{l}\right)^{2}}-1\right)^{\frac{1}{2}}
$$

The metric is then determined uniquely by $q$ and $r_{0}$; given the restriction $r_{0}>r_{c}$, the mass is totally fixed:

$$
m=\frac{r_{0}}{2}\left(\frac{r_{0}^{2}}{l^{2}}+\frac{q^{2}}{r_{0}^{2}}+1\right)
$$

All properties of the Euclidean section are then functions of $r_{0}$ and $q$. In particular, the period $\beta_{R N}$ is given as

$$
\beta_{R N}=\frac{4 \pi r_{0}}{\frac{3 r_{0}^{2}}{l^{2}}-\frac{q^{2}}{r_{0}^{2}}+1}
$$


We now have all the ingredients necessary to apply the 'no-boundary proposal' to the calculation of the rate of the black hole pair production in these non-extreme SUGRA domain wall backgrounds. For simplicity, we begin with the case where the cosmological constant is the same on each side of the wall $\left(\Lambda_{1}=\Lambda_{2}=\Lambda\right)$; we will then make some general comments about the non-symmetric $\left(\Lambda_{1} \neq \Lambda_{2}\right)$ situation.

Case $1\left(\Lambda_{1}=\Lambda_{2}=\Lambda\right)$

Here, we just need to determine the values of $m$ and $q$ (or equivalently, $r_{0}$ and $q$, we have fixed $\Lambda$ ) for which we can satisfy the following two relations simultaneously:

$$
[C]\left\{\begin{array}{c}
m>m_{c}(q) \\
\beta_{W}=\frac{\beta_{R N}}{n}, n \in \mathbb{Z}_{+}
\end{array}\right.
$$

The line integral for $\beta_{W}$ must be performed numerically. Below, we plot the two periods $\beta_{W}$ and $\beta_{R N}$, as functions of charge $q$; the range of q runs from zero (where we recover Schwarzschild-adS black holes) to an upper bound, $q_{\max }$, determined by the non-degeneracy condition $m>m_{c}(q)$ : Fig. 2

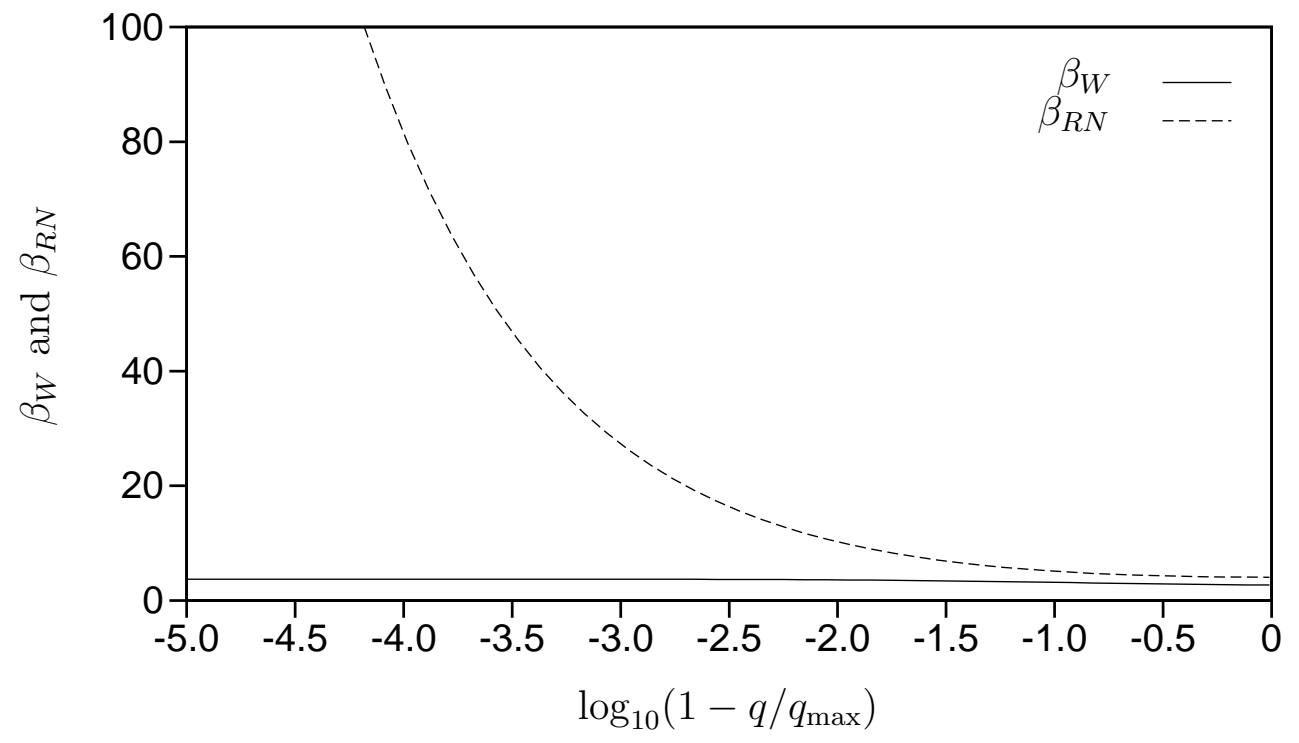

What this figure tells us is clear: For given values of the energy density of the 
wall (here, $\sigma=\frac{1}{2 \pi} \sqrt{\frac{|\Lambda|}{3}+\beta^{2}}$ ) and the mass $m$, there exists a countably infinite set of charges $\left\{q_{n}\right\}$, for which it is possible to find instantons satisfying the constraints $[C]$ (above) which will mediate the creation of accelerating black hole pairs from the $\mathbb{Z}_{2}$-symmetric initial domain wall state.

Finally, we point out that as usual there exists a 'static limit' domain, i.e., $\dot{r}=0$. This static wall lies at $r_{s}$, which is calculated to be $r_{s}=\frac{3}{2} m\left[1+\sqrt{1-\frac{8}{9} \frac{q^{2}}{m^{2}}}\right]$

As usual, we calculate the rate of black hole pair creation by dividing the amplitude to create the combined black hole-domain wall configuration by the amplitude to create just the domain wall configuration. In each case, the amplitude is given as

$$
\mathcal{A}=e^{-\mathcal{S}}
$$

where $\mathcal{S}$ is half the action of the Euclidean section of the relevant solution.

In general, the action in Einstein-Maxwell theory with a negative cosmological constant and a domain wall boundary term is written as

$$
\mathcal{S}=\frac{1}{16 \pi} \int_{M_{e}} d^{4} x \sqrt{g}\left(R-2 \Lambda-F^{2}\right)+\frac{1}{2} \sigma \int_{W} \sqrt{h} d^{3} x
$$

where $R$ is the four-dimensional Ricci scalar, $\sigma$ is the energy density of the wall and

$$
\int_{W} \sqrt{h} d^{3} x=\operatorname{vol}(W)
$$

is the volume of the closed, three-dimensional surface determined by the domain wall on the Euclidean section.

We begin by calculating the action of the Euclidean section of the non-extreme, $\Lambda_{1}=\Lambda_{2}=\Lambda$ domain wall solution. Here,

$$
\begin{array}{r}
\sigma=\frac{1}{2 \pi} \sqrt{\frac{|\Lambda|}{3}+\beta^{2}} \\
F^{2}=0
\end{array}
$$

Since each side of the wall is a portion of adS,

$$
R=4 \Lambda
$$

The Euclidean section, $M_{e}$, is obtained by glueing two hyperbolic four-balls together along their boundary three-spheres; one then sees the $M_{e}$ is topologically $S^{4}$, only 
with a 'ridge' of curvature along the domain wall $W=S^{3}$. Thus, it simply remains to determine $\operatorname{vol}\left(M_{e}\right)$ and $\operatorname{vol}(W)$.

To calculate these quantities, first recall [15] that the volume of a sphere of geodesic radius $r$ in hyperbolic space of constant negative sectional curvature $\kappa$, is given as

$$
\operatorname{vol}\left(S^{3}(r)\right)=2 \pi^{2}\left(\frac{1}{\sqrt{|\kappa|}} \sinh (\sqrt{|\kappa| r})\right)^{3}
$$

Now, on a four-manifold $R=4 \kappa$, and so $\Lambda=\kappa$ in our case; also, in the above we are working in 'exponential' coordinates, so that $r$ is the coordinate obtained by projection of the usual radial coordinate on $\mathbb{R}^{4}$ (recall that the map exp : $\mathbb{R}^{4} \longrightarrow H_{\kappa}^{4}$, where $H_{\kappa}^{4}$ is hyperbolic space, is in fact a global diffeomorphism since the injectivity radius of $H_{\kappa}^{4}$ is infinity).

Given Equation (15) for the volume of a three-sphere of radius $r$ in $H_{\Lambda}^{4}$, it is then obvious that the volume of a ball of radius $r$ is given as

$$
\operatorname{vol}\left(B^{4}(r)\right)=2 \pi^{2} \int_{0}^{r}\left(\frac{1}{\sqrt{|\Lambda|}} \sinh (\sqrt{|\Lambda| r})\right)^{3} d r
$$

Since $M_{e}$ is obtained by glueing two of these balls together, we need only ask: what is the radius, $r$ ?

The radius of the balls is determined by the matching condition for joining the instanton to the Lorentzian section; in particular, we must match the Euclidean and Lorentzian sections along a spacelike three-surface of vanishing extrinsic curvature (so that we can think of the tunneling process in terms of a 'path' of spacelike threegeometrics). In other words, we match the two sections along the surface where the wall is (instantaneously) stationary. Since the wall is simply moving with uniform acceleration in adS, this surface is not too difficult to locate. The most transparent way to do the calculation is to map the adS coordinates used above to the Einstein cylinder coordinates (recall that adS is conformalm to 'one half' the Einstein static universe [17]). In terms of the coordinates $(t, z, r, \phi)$, the new coordinates are

$$
\begin{aligned}
& T=\xi \sinh (\beta t) \\
& R=\xi \cosh (\beta t)
\end{aligned}
$$


where $\ln \xi=\beta\left(z-z^{\prime}\right)$. The domain wall (at $z=0$ ) then lives on the hyperbolic trajectory [16]

$$
R^{2}-T^{2}=\frac{1}{\delta}
$$

where

$$
\delta=\frac{\frac{|\Lambda|}{3}+2 \beta^{2}+2 \beta\left(\beta^{2}+\frac{|\Lambda|}{3}\right)^{\frac{1}{2}}}{\frac{|\Lambda|}{3}}
$$

The wall is stationary at $T=t=0$, and so the 'critical' value $\left(R_{c}\right)$ of the radius is given by

$$
R_{c}=\frac{1}{\beta}
$$

Where this is the radius measured in the metric of hyperbolic space, i.e., the metric induced by the Gaussian normal coordinates, and so $R_{c}$ is the actual geodesic radius of the instanton.

The volume calculation is time-consuming and yields

$$
\begin{aligned}
& \frac{1}{2} \operatorname{vol}\left(M_{e}\right)=2 \pi^{2} \int_{0}^{R_{c}}\left(\frac{1}{\sqrt{|\Lambda|}} \sinh (\sqrt{|\Lambda| r})\right)^{3} d r \\
= & \frac{4 \pi^{2}}{(\sqrt{|\Lambda|})^{5}}\left\{\frac{3}{4} \sinh \sqrt{|\Lambda| R_{c}}-\frac{3}{4} \sqrt{|\Lambda| R_{c}} \cosh \sqrt{|\Lambda| R_{c}}\right. \\
& \left.-\frac{1}{36} \sinh \left(3 \sqrt{|\Lambda| R_{c}}\right)+\frac{\sqrt{|\Lambda| R_{c}}}{12} \cosh \left(3 \sqrt{|\Lambda| R_{c}}\right)\right\}
\end{aligned}
$$

The volume of the wall is just the volume of a three-sphere of radius $R_{c}$ :

$$
\operatorname{vol}(W)=\frac{2 \pi^{2}}{(\sqrt{|\Lambda|})^{3}} \sinh ^{3}\left(\sqrt{|\Lambda| R_{c}}\right)
$$

Thus the total action, $\mathcal{S}_{W}$, for the Euclidean section of the $\mathbb{Z}_{2}$-symmetric nonextreme domain configuration is

$$
\frac{1}{2} \mathcal{S}_{W}=\frac{\Lambda}{8 \pi} \operatorname{vol}\left(M_{e}\right)+\frac{\pi^{2} \sigma}{2(\sqrt{|\Lambda|})^{3}} \sinh ^{3}\left(\sqrt{|\Lambda| R_{c}}\right)
$$

We now just need to calculate the action, $\mathcal{S}_{R N}$, of the Euclidean section of the $\mathbb{Z}_{2}$-Symmetric RNadS-domain wall configuration. We have to deal with the extra $F^{2}$ term. As usual, we can either consider electrically charged holes, for which

$$
F=-\frac{q}{r^{2}} d t \wedge d r
$$


or magnetically charged holes, for which

$$
F=q \sin \theta d \theta \wedge d \varphi
$$

In this paper we shall restrict our attention to the creation of magnetically charged holes; by the usual duality arguments [18] we expect the rate of electric hole production to be the same (ignoring the 1-loop effects of any matter fields, which obviously would violate electric-magnetic duality).

We begin by calculating the probability to create static holes, i.e., holes whose attractive gravitational energy exactly counterbalances the repulsive energy of the wall. Thus, as we saw above, the black holes will lie at radius $r_{s}$ from the wall, where

$$
r_{s}=\frac{3}{2} m\left[1+\sqrt{1-\frac{8}{9} \frac{q^{2}}{m^{2}}}\right]
$$

The mass, $m$, is completely determined in terms of $q$ and $\Lambda$ (using relations (10)-(11) above). Now, as discussed above, in order for the motion of the wall on the black hole instanton to be consistent, the mass $m$ is further constrained to lie in the interval $\frac{1}{3 \sqrt{3} \beta} \leq m \leq \frac{1}{4 \beta}$. Thus, as the acceleration parameter $\beta$ is increased, the mass is forced to decrease (as is $r_{s}$, in light of Equation 23).

The action calculation is now much more straightforward. Let $r_{h}$ denote the outer horizon radius as above. Then the volume contribution is

$$
\frac{1}{16 \pi} \int_{B_{e}}\left(2 \Lambda-F^{2}\right) \sqrt{g} d^{4} x=\beta_{R N} \Lambda\left[\frac{r_{s}^{3}-r_{h}^{3}}{12}\right]+\frac{q^{2}}{4} \beta_{R N}\left(\frac{1}{r_{h}}-\frac{1}{r_{s}}\right)
$$

where $B_{e} \cong D^{2} \times S^{2}$ is one-half of the Euclidean section of the black hole-domain wall configuration.

The domain wall term is

$$
\sigma \int_{W} \sqrt{h} d^{3} x=4 \pi \sigma r_{s}^{2} \beta_{R N} \sqrt{f\left(r_{s}\right)}
$$

Thus, the total action $\mathcal{S}_{R N}$ is given as

$$
\frac{1}{2} \mathcal{S}_{R N}=\beta_{R N} \Lambda\left[\frac{r_{s}^{3}-r_{h}^{3}}{12}\right]+\frac{q^{2}}{4} \beta_{R N}\left(\frac{1}{r_{h}}-\frac{1}{r_{s}}\right)+4 \pi \sigma r_{s}^{2} \beta_{R N} \sqrt{f\left(r_{s}\right)}
$$


The probability, $P$, that static black holes of charge $\pm q$ and mass $m$ will be created in the presence of the $\mathbb{Z}_{2}$-symmetric wall background is then given as

$$
P=\exp \left(\mathcal{S}_{W}-\mathcal{S}_{R N}\right)
$$

where $\mathcal{S}_{W}$ and $\mathcal{S}_{R N}$ are given in equations (22) and (26) respectively.

$P$ is plotted below, for fixed $\Lambda, m$, and $\beta$ (and hence $\sigma$ ) as $q \longrightarrow q_{\max }$ : Fig.3

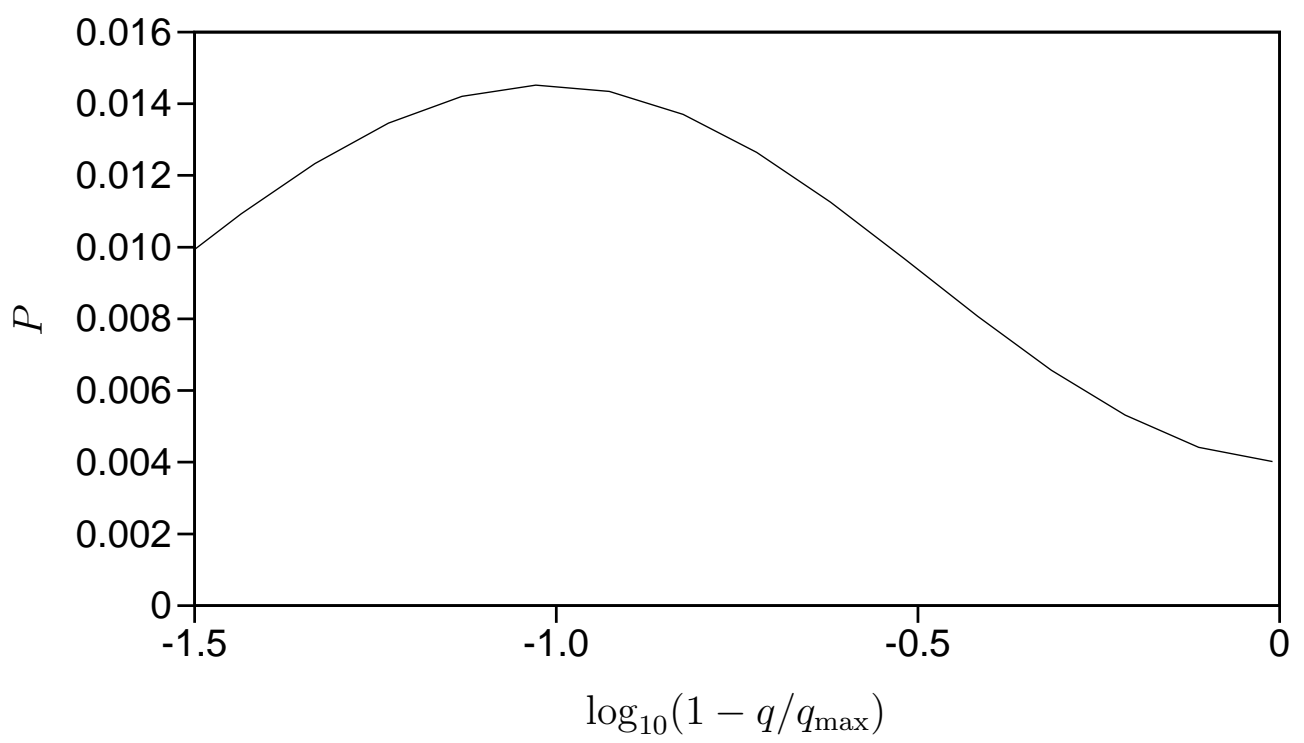

To calculate the rate of production of oppositely charged, accelerating black hole pairs in the $\mathbb{Z}_{2}$-symmetric background, we first restrict the charge to be equal to one of the values $\left\{q_{n}: n \in \mathbb{Z}_{+}\right\}$such that, for fixed $m, \Lambda$, and $\sigma$, the two periods $\beta_{W}$ and $\beta_{R N}$ satisfy the matching condition

$$
\beta_{W}=\frac{\beta_{R N}}{n}, n \in \mathbb{Z}_{+}
$$

As we saw above in Fig. 2, the $q_{n}$ always exist for generic $m$ and $\Lambda$. We therefore simply need to calculate $\mathcal{S}_{R N}$ for the Euclidean section of the accelerating black holedomain wall configuration. As above, $\mathcal{S}_{R N}$ decomposes into a 'volume' part and a 'domain wall' part. The calculation is a straightforward generalision of the results of [7] and yields

$$
\frac{1}{2} \mathcal{S}_{R N}=2 \pi \sigma \int_{r_{\min }}^{r_{\max }} d r \frac{2 \pi \sigma r^{3}}{\sqrt{f\left(f-(2 \pi \sigma r)^{2}\right)}}
$$




$$
\begin{gathered}
+\frac{q_{n}^{2} \beta_{R N}}{4 r_{h}}-\frac{q_{n}^{2}}{2} \int_{r_{\min }}^{r_{\max }} d r \frac{2 \pi \sigma}{f \sqrt{f-(2 \pi \sigma r)^{2}}} \\
-\frac{\Lambda \beta_{R N} r_{h}^{3}}{12}+\frac{\Lambda}{6} \int_{r_{\min }}^{r_{\max }} d r \frac{2 \pi \sigma r^{4}}{f \sqrt{f-(2 \pi \sigma r)^{2}}}
\end{gathered}
$$

Thus, the probability that accelerating black hole pairs of mass $m$ and opposite charge $\pm q_{n}$ will be nucleated in the presence of a $\mathbb{Z}_{2}$-symmetric wall configuration is given by eq. 27 , where now $\mathcal{S}_{R N}$ is given by equation (28).

$P$ is again plotted below, for fixed $\Lambda, m$ and $\beta$ as $q \longrightarrow q \max$ : Fig. 4 .

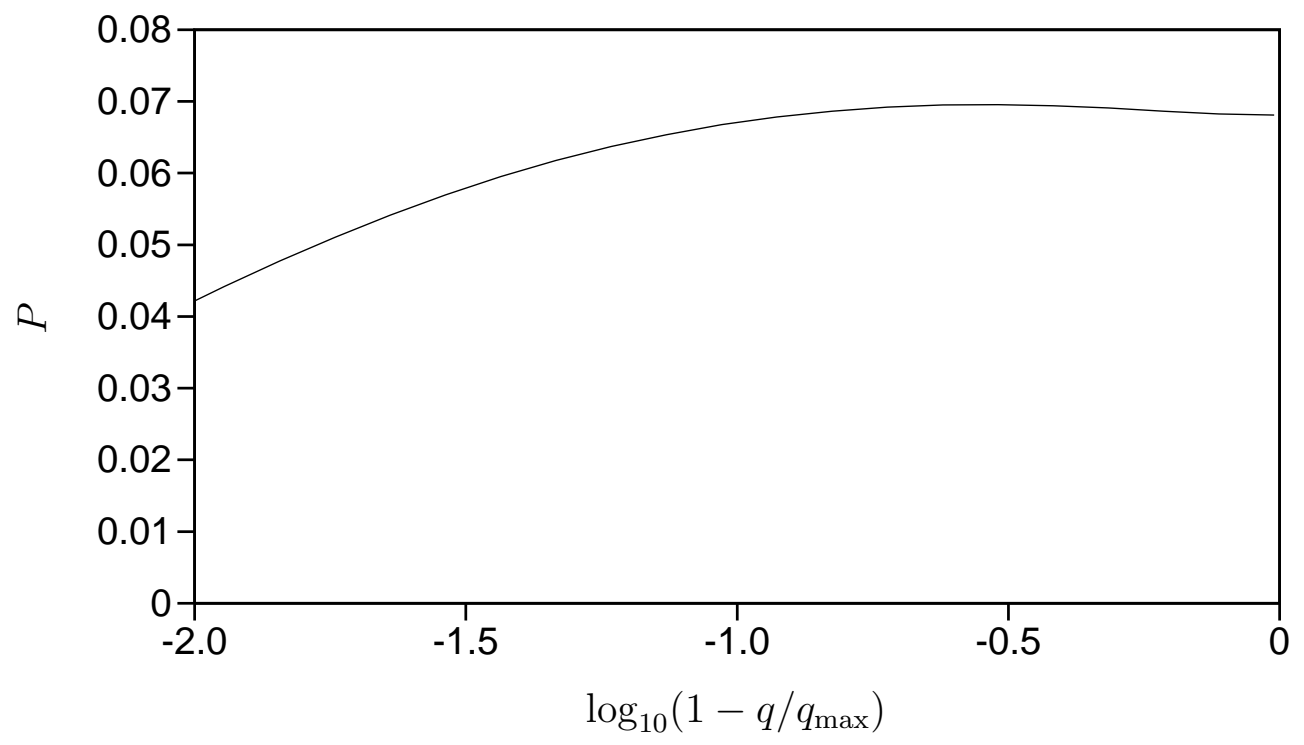

The above calculations were carried out for the values $\Lambda=-1, m=\frac{1}{3 \sqrt{3}}$, and $\beta=1$; however, the basic properties of the probability distributions shown are generic for all values of these parameters. In particular, the rate of creation is always suppressed as the charge approaches the extremal limit.

Case $2\left(\Lambda_{1} \neq \Lambda_{2}\right)$

In the most general situation where the cosmological constant varies as we move across the domain wall, we would still expect the configuration to be unstable to a semiclassical process such as black hole pair creation; after all, the only selection rule we need to worry about is charge conservation. The only thing exotic about 
these non-symmetric walls is that the magnitude of repulsion will depend on which side you are on. Naively, we would therefore expect that black holes of equal and opposite charge, but unequal mass, would be created in the presence of these walls.

However, there is a simple geometrical obstruction to the construction of regular instantons for these configurations; in fact, it would seem that it is not possible to obtain a well behaved Riemannian section for the domain wall, much less for the full black hole-domain wall situation.

We again attempt to construct the instanton for the domain wall by taking two hyperbolic four-balls, each of radius $\frac{1}{\beta}$, but of unequal constant negative curvatures $\Lambda_{1}$ and $\Lambda_{2}$. We have to be able to join these two objects along their respective boundaries in order to obtain a 'nice' instanton $M_{e}$ of topology $S^{4}$ with a ridge of curvature running along where the domain wall is. However, equation (15) for the volume of a three-sphere of radius $r$ in hyperbolic space makes clear that the volumes of the boundaries of the two balls will match if and only if the two cosmological constants are equal! In other words, the two balls will be rather like a large plate and a small bowl; the plate will always fit 'over' the rim of the bowl, but it is impossible to align them along their respective edges. While one might devise various clever schemes to get around this pathology, we will have nothing more to say about these non-symmetric walls in this paper.

\section{No Pair Production of Black Holes by Extreme Vacuum Walls}

As we allow the acceleration parameter $\beta$ to approach zero, the size of the wall tends to infinity until finally, when extremality is reached, the wall 'decompactifies' and turns into a static copy of $2+1$ Minkowski space. However, as we have seen the resulting extreme configuration is still repulsive; hence, one might expect the repulsive energy to provide a source for black hole pair production.

On the other hand, the radius of the instanton obtained in the extremal limit is infinite, and since the volume contribution to the action (eq. (22)) will always 
dominate for large $r$, the domain wall action $S_{W}$ is negative infinite. Thus, in order for the process to be unsuppressed there would have to be a term in the action $S_{R N}$ which effectively counterbalanced the large volume term coming from $S_{W}$. The only candidate term in $S_{R N}$ which could play such a role is the volume term proportional to $\Lambda$. However, this term only grows as $r_{w}^{3}$, where $r_{w}$ is the 'size' of the wall (recall that this is all consistent since, for very small $\beta, r_{w} \sim \frac{1}{\beta}$ ), whereas the dominant volume term coming from $S_{W}$ grows like

$$
\int_{0}^{\frac{1}{\beta}}\left(\frac{1}{\sqrt{|\Lambda|}} \sinh (\sqrt{|\Lambda| r})\right)^{3} d r
$$

Thus, it is clear that the term which is driving $S_{W}$ to minus infinity will always dominate and so the rate will be competely suppressed in the extremal limit. Indeed, one can see this effect numerically; as $\beta$ gets smaller and smaller the probability contours 'collapse' down towards zero, until finally the plot of $P$ looks effectively like $P \sim 0$ for all allowed values of the charge.

Of course, this effect would be expected on the grounds that as one approaches the extremal state one is approaching a BPS state which should be stable to all quantum corrections. This is reminiscent of the situation of black hole pair production in the early universe [19]; there, as one turns down the cosmological constant and approaches the stable Minkowski vacuum state the rate of black hole pair creation is totally suppressed in the limit. The difference in our example is that the SUSY state which is obtained in the limit is much more complicated and has a richer causal structure than that of Minkowski space [1].

\section{Conclusions and Discussion}

We have shown that non-extreme vacuum domain walls which arise in $N=$ $1, d=4$ supergravity are unstable to black hole pair creation; furthermore, we found that the production rate goes to zero as the walls are allowed to approach extremality. This work is an interesting generalization of the work presented in [7]. 
The global causal structure of the SUGRA domain walls studied here is much more subtle than that of the simpler VIS walls discussed in [7], and furthermore such objects are simple prototypes of the 'braney' type solutions which arise commonly in the supergravity menagerie.

Of course, a key ingredient of Kaluza-Klein, supergravity, and effective theories derived from string theory is the dilaton. It is therefore of considerable interest to consider the semi-classical stability of domain walls which are coupled to the dilaton. Such dilatonic walls have been studied for some time, and there is an extensive literature about them (see [1] for an overview). As would be expected (from the example of dilatonic black holes), turning on the dilaton coupling changes the global spacetime structure of the solutions. In fact, in the non-extreme case these walls often exhibit naked singularities; this was one of our motivations for not considering these walls in this paper. Nevertheless, in any regime where one can find non-extreme configurations which are well-behaved we would expect the basic results of this paper to go through, i.e., Reissner-Nordström-dilaton anti-de Sitter black holes will be produced by the non-extreme backgrounds, and the rate of production will go to zero as extremality is approached. On a related note, it should also be of interest to see what happens when the walls are coupled to gauge fields; with the introduction of gauge charges it should be possible to attain extremality without resorting to a cosmological constant.

It is worth pointing out that there is a well-defined procedure by which these solutions can be obtained via dimensional reduction of higher dimensional configurations in supergravity (or string theory); in particular, in [20] it was shown that the dilatonic domain wall configurations of Cvetič et al could be obtained by performing Scherk-Schwarz dimensional reduction on higher dimensional supergravity solutions. The Scherk-Schwarz procedure, which was originally introduced to break SUSY by giving mass to the gravitino, in the present case means that some of the fields (the axions) are allowed to have a linear dependence on a compactification coordinate. This dependence leads to a cosmological constant term in the lower dimensional, massive supergravity theory. The $(d-2)$-brane solutions of the massive 
supergravity in four dimensions are precisely the dilatonic walls discovered earlier.

Of course, it is well known that black hole solutions can also be obtained by taking the ordinary Kaluza-Klein reduction of certain higher dimensional configurations in string theory. The fact that both types of object, black hole and domain wall, naturally oxidize to higher dimensional string configurations suggests that there might be an 'oxidation' of the semi-classical tunneling process of black hole pair creation to higher dimensions. Of course, one has to be careful, since at least some of the domain wall energy density (the part proportional to the cosmological constant) is coming from the compactification process. These problems, and many more, are currently being actively investigated.

\section{Acknowledgements}

We wish to thank R. Caldwell and R. Emparan for useful discussions. A.C. was supported by NSF PHY94-07194, and J.M.A.A-C was supported by Wolfson College, University of Oxford.

\section{References}

[1] M. Cvetič and H.H. Soleng, Phys. Rep. 282, 159-223 (1997), preprint hep-th/9604090 (1996).

[2] M. Cvetič and S. Griffies, Phys. Lett. B 285 (1992) 27.

[3] M. Cvetič, S. Griffies and S.J. Rey, Nucl. Phys. B 381 (1992) 301.

[4] M. Cvetič, S. Griffies and H.H. Soleng, Phys. Rev. D 48 (1993) 2613.

[5] M. Cvetič, S. Griffies and H.H. Soleng, Phys. Rev. Lett. 71 (1993) 670. 
[6] M. Cvetič, S. Griffies and S.J. Rey, Nucl. Phys. B 389 (1993) 3.

[7] R.R. Caldwell, A. Chamblin and G.W. Gibbons, Phys. Rev. D 53 (1996) 7103.

[8] G.W. Gibbons, Nucl. Phys. B 395 (1993) 3.

[9] G.W. Gibbons, Nucl. Phys. B 472 (1996) 683.

[10] M. Cvetič and S. Griffies, Phys. Lett. B 285 (1992) 27.

[11] R.B. Mann, preprint gr-qc/9607071 (1996).

[12] J. Ipser and P. Sikivie, Phys. Rev. D 30 (1984) 712.

[13] S.J. Poletti, J. Twamley and D.L. Wiltshire, Phys. Rev. D 51 (1995) 5720.

[14] J. Louko and S.N. Winters-Hilt, Phys. Rev. D 54 (1996) 2647.

[15] I. Chavel, Riemannian Geometry - A Modern Introduction, CUP, Cambridge (1993).

[16] M. Cvetič, preprint hep-th/9306015 (1993).

[17] S.W. Hawking and G.F.R. Ellis, The Large-Scale Structure of Spacetime, CUP, Cambridge (1973).

[18] S.W. Hawking and S.F. Ross, Phys. Rev. D 52 (1995) 5865.

[19] P. Ginsbarg and M.J. Perry, Nucl. Phys. B 222, 245 (1983).

[20] P.M. Cowdall, H. Lu, C.N. Pope, K.S. Stelle and P.K. Townsend, Nucl. Phys. B 486, 49-76 (1997). 
